\title{
Insecticide Treated Net (ITN) Utilization and Associated Factors among Pregnant Women's in Halaba Kulito Town, South Ethiopia, 2016: Cross-Sectional Study
}

\author{
Alex Yeshaneh", Daniel Adane \\ Department of Midwifery, College of Medicine and Health Sciences, Wolkite University, Wolkite, Ethiopia \\ Email address: \\ alexyeshaneh7@gmail.com (A. Yeshaneh) \\ ${ }^{*}$ Corresponding author \\ To cite this article: \\ Alex Yeshaneh, Daniel Adane. Insecticide Treated Net (ITN) Utilization and Associated Factors among Pregnant Women's in Halaba Kulito \\ Town, South Ethiopia, 2016: Cross-Sectional Study. International Journal of Infectious Diseases and Therapy. \\ Vol. 5, No. 2, 2020, pp. 34-40. doi: 10.11648/j.ijidt.20200502.13
}

Received: July 2, 2019; Accepted: August 4, 2019; Published: May 29, 2020

\begin{abstract}
Background:-Malaria remains to be a major problem causing an unacceptable toll on the health and economic welfare of world's poorest communities. Globally, it is agreed to reduce the burden of malaria by half by the year 2010 and again by half by 2015. Similarly, at the African summit on Roll Back Malaria (RBM) in Abuja, committed themselves to halving the burden of malaria by 2010 , by achieving $60 \%$ coverage of all at risk population with suitable curative and preventive measures by 2005. Ethiopia, as one of the signatories of the Abuja convention, has included the above global strategies in its national malaria prevention and control approach. Therefore, the aim of this study was to assess Insecticide Treated Nets utilization and associated factors among pregnant women in Halaba kulito town, 2016. Methods: A community based cross sectional study was carried out among pregnant women in Halaba Kulito town. The study included 92 mothers who were selected by simple random sampling. Household survey using structured questionnaire was used to collect information. Logistic regression analyses were employed to identify factors associated with ITN utilization. Variables with a pvalue $<0.05$ were identified as statistically significant factors. Results: Only $51(70.8 \%)$ of pregnant women had self-reported as utilizing bed net during in previous night of survey. The direct observation done had shown that only 39 (42.4\%) of pregnant women were directly observed while sleeping under bed net. Among this $19(20.43 \%)$ of pregnant women's properly utilize mosquito net. Conclusion: The time taken to reach health institution, the type of bed room condition and level of education were some of significant factors which were associated with ITN Utilization. Majority of the pregnant women did not utilize ITN. Therefore, the free distribution should prioritize vulnerable groups using good opportunity of Antenatal care clinic, household education and regular district level on going survey.
\end{abstract}

Keywords: ITN Utilization, Pregnant, Ethiopia

\section{Introduction}

Globally, Malaria remains to be a major problem causing an unacceptable toll on the health and economic welfare of world's poorest communities. World Health Organization report has shown that the disease is estimated to be responsible for an estimated average annual reduction of $1.3 \%$ in economic growth for those countries with the highest burden. Africa has the greatest burden of malaria cases and deaths in the world. The effects of malaria in pregnancy are enormous and include maternal anemia, intrauterine growth restriction, low birth weight, and premature deliveries among others. Malaria is also a significant indirect cause of death; malaria related maternal anemia in pregnancy, low birth weight and premature delivery are estimated to cause 75,000200,000 infant deaths per year in Africa South of the Sahara $[1,2]$. Malaria transmission in Ethiopia is mostly seasonal and unstable in characteristics, thus, predisposing majority of the population to frequent and often large-scale epidemics. Parasite species of all the four Plasmodium species occurring in the country, the two epidemiologically important species are Plasmodium falciparum and Plasmodium vivax, $60 \%$ and 
$40 \%$, respectively. Anopheles arabinoses is the principal vector adapted to different ecological locations in Ethiopia [3].

Despite the highest magnitude of this devastating disease, most countries did not start implementing program to provide access to the tools and strategies recommended by Roll Back Malaria until 2000. Globally, it is agreed to reduce the burden of malaria by half by the year 2010 and again by half by 2015. In many countries in Africa where the burden of malaria is greatest, scaling up access to treatment and prevention began even more recently [1]. For the unborn child, maternal malaria increases the risk of spontaneous abortion, stillbirth, premature delivery, low birth weight and intrauterine growth restriction a leading cause of child mortality [4]. Malaria is a major public health problem in tropical and subtropical regions of the world. The World Health Organization (WHO) 2011 World Malaria Report demonstrates the enormity of the burden of malaria, with 216 million cases and 655,000 deaths attributable to this mosquito-transmitted parasite in 2010 alone. The burden is largely borne by Africa where $91 \%$ of deaths occurred, with pregnant women most at risk of infection and adverse outcomes. Each year, there are an estimated 25 million pregnancies in sub-Saharan Africa at risk of malaria, the consequences of which can be serious for both mother and fetus in terms of morbidity and mortality [1].

In Africa, 30 million women living in malaria-endemic areas become pregnant each year. For these women, malaria is a threat both to themselves and to their babies, with up to 200000 newborn deaths each year because of malaria in pregnancy. Pregnant women are particularly vulnerable to malaria as pregnancy reduces a woman's immunity to malaria, making her more susceptible to malaria infection. It increases the risk of illness, severe anemia, hypoglycemia, pulmonary edema, coma and death. They are more likely to develop cerebral and other forms of severe malaria, and suffer a high mortality 2 to 10 times higher than nonpregnant patients. They are particularly also susceptible to hypoglycemia and acute pulmonary oedema. Falciparum malaria commonly induces uterine contractions and gives rise to premature labor [5]. Sleeping under ITNs remains an important strategy for protecting pregnant women and their newborns from malaria-carrying mosquitoes. In areas of low or unstable malaria transmission, pregnant women have low immunity to malaria and a two- to threefold higher risk of severe malarial illness than non-pregnant women. In these areas, use of ITNs and prompt case management of pregnant women with fever and malarial illness are the main strategies for malaria prevention and treatment [6].

Sub-Sahara Africa harbor's $80-90 \%$ of the world malaria burden and about 19-24 million women are at risk of malaria during pregnancy. It contributes up to $11 \%$ of maternal mortality in the region. Assessment of attaining of Abuja target of $60 \%$ ITNs coverage by African countries indicated that Eritrea is the only one that reached this target during 2003 (WHO, 2005). Malaria is one of the top ranking causes of morbidity and mortality in Ethiopia. The highest score for outpatient visit goes for malaria. It is also one of the main causes of hospitalization Moreover, death in all corners of the country. More than two-thirds of population lives in malarious areas [7, 8]. In 2000-2003, the disease was the primary cause of reported morbidity and mortality accounting for $16 \%$ of outpatient visit, $20 \%$ of hospital admission, and $27 \%$ of hospital deaths. During a nonepidemic years, 5-6 million clinical malaria cases and over half a million confirmed cases were reported from health facilities [8]. Ethiopia, realizing the effectiveness of ITNs for prevention of malaria transmission, scaling up distribution and utilization of ITN to cover $60 \%$ of pregnant women living at high risk area of malaria transmission is undergoing. In order to support this part of malaria prevention aspect there was much effort and resources allocated from donors. On the other hand, the proper utilization coverage of ITNs among priority and high-risk groups were found to be very low, whereas the $1^{\text {st }}$ phase of the commitment has ended by 2005 [4].

\section{Methods}

\subsection{Study Setting and Design}

A community based cross-sectional study was conducted from April 15-20, 2016 at Halaba Kulito town, which is located $315 \mathrm{~km}$ far from Addis Ababa and $90 \mathrm{~km}$ southwest of Hawassa. The Town is administratively divided in to two 'kifle-ketemas' (sub-towns) and five 'kebeles' all of which were malarias'. The town has an estimated total population size of 37,689 . From these, 17,845 were males and 19,844 were females and it has one health center and one primary hospital.

\subsection{Populations}

\subsubsection{Source Population}

A pregnant woman's who were registered by health extension workers of Halaba Kulito town.

Study population

Pregnant mothers who was included in sampling size during the data collection period.

\subsection{Inclusion and Exclusion Criteria}

Pregnant women, who were selected in sample size and residents of Halaba Kulito town for greater than or equal to six months were included. Pregnant women's who were mentally and critically ill and not able to speak at the time of data collection were excluded.

\subsection{Sample Size Determination}

The study sample size was determined by statistical calculation. The estimation of population proportion, $p$, where $p$ is the proportion of households that uses ITNs for pregnant women, $\mathrm{p}=0.147$ was used which was done in Adama woreda [9], as this value gives sample size sufficiently large to guarantee an accurate prediction, at $95 \%$ 
confidence interval and $5 \%$ error of estimate. The following formula was used $n=(Z \alpha / 2) 2 \times p(1-p) / d^{2}$ whereas: $\mathrm{n}=$ sample size $\mathrm{p}=0.147$ (proportion of pregnant women using ITNs) $Z \alpha / 2=1.96$ ( $Z=$ score corresponds to $95 \%$ confidence interval. $d=0.05$ (margin of error). Total sample size was 193 . Since the source population is less than determined sample size, we have used reduction formula i.e. $n /(1+n / N)=192 /$ $(1+192 / 180)=93$ pregnant women's.

\subsection{Sampling Technique}

All kebeles were included and the study participants were selected by simple random sampling method with proportion from each Kebele using the registration list, which was prepared by health extension workers of the town.

\subsection{Operational Definition}

Bed Net-is a material made of nylon, polyester, polyethylene and synthetic with cotton mixture with different shape, size and used to protect people against insect bites.

Insecticide Treated Mosquito Nets- Nets treated with insecticide to kill or irritate mosquitoes and used as physical barriers.

ITN utilization- The use of standardized properly hanged (mounted) over the bed or the sleeping area, no thorn /without defect and pregnant women sleeping under the mosquito net during the early morning of observation day.

\subsection{Data Collection Tools and Procedures}

Data were collected by using semi-structured questionnaires. Both closed ended and open-ended questions were utilized for data collection. The questionnaire initially was prepared in English and then translated to local language Amharic then back to English version to ensure consistency. The questionnaires include items informed consent of respondent and questions. Five midwifery students were collected data on daily basis from morning to evening including weekends for the period of two weeks starting from April 15-20, 2016. The questionnaire took 20-30 minutes to interview one-study participants.

\subsection{Data Entry and Analysis}

The data was cleaned, coded, entered, and analyzed using SPSS version 20. Descriptive statistics was computed to assess Utilization of Insecticide Treated Nets and association factors. Logistic regression analysis was used to obtain odds ratio and the confidence interval of statistical associations. The strength of statistical association was measured by adjusted odds ratios and $95 \%$ confidence intervals. Statistical significance was declared at $\mathrm{P}<0.05$. finaly finding of the result was presented by text, figure, graphs and tables.

\subsection{Data Quality Control}

Investigators were reviewed all the questionnaires, checked for errors and incompleteness at the end of each day. Five data collectors (midwifery students) were participated in data collection. Information was given for data collectors on how to collect data, on the objectives of the study, the contents of the questionnaire, and particularly on issues related to the confidentiality of the responses and the rights of respondents. The Questionnaires were checked for completeness on daily bases and take appropriate measures. One week prior to data collection pretest was conducted on $5 \%$ of women and based on the finding necessary modification was made before conducting the actual study and those participated in the pretest was excluded from actual study.

\section{Result}

\subsection{Socio-demographic Characteristics of Households}

A total of $93(100 \%)$ study subjects were participated in the study. Out of these participants $35(37.6 \%)$ respondents were in 25 to 29 years age group and $90(96.8 \%)$ were married. From total of study participants $35(37.6 \%)$ had secondary education, 35 (37.6\%) were Muslims, 35 (37.6\%) were housewives and $31(33.3 \%)$ were Kambata by ethnicity (Table 1).

Table 1. Socio-demographic characteristics of pregnant women's in Halaba Kulito town, 2016.

\begin{tabular}{lll}
\hline Age of respondents & Frequency & Percent (\%) \\
\hline $20-24$ & 33 & 35.5 \\
$25-29$ & 35 & 37.6 \\
$30-34$ & 12 & 12.9 \\
$\geq 35$ & 13 & 14 \\
Ethnicity of respondent & & \\
Halaba & 29 & 31.2 \\
Kambata & 31 & 33.3 \\
Others & 33 & 35.5 \\
Marital status of the respondent & & \\
Married & 90 & 96.8 \\
Non married & 3 & 3.2 \\
Religion of respondents & & \\
Orthodox & 23 & 24.7 \\
Muslim & 35 & 37.6 \\
Protestant & 32 & 34.4 \\
Others & 3 & 3.2 \\
Occupation of respondents & & \\
Government employer & 15 & 16.2 \\
Private worker & 19 & 20.4 \\
Merchant & 24 & 25.8 \\
Housewife & 35 & 37.6 \\
Educational status the respondents & & \\
No education & 20 & 21.5 \\
Primary education & 17 & 18.3 \\
Secondary education & 35 & \\
More than secondary education & 21 & \\
\hline & & \\
\hline
\end{tabular}

\subsection{ITN Coverage and Conditions Related to Pregnant Women's Net}

A total of $79(84.95 \%)$ of the respondents reported that they have their own at least one bed net.

But, $7(8.86 \%)$ pregnant women's couldn't show any bed net during observation of households. Among household's bed nets which were observed, 18 (25\%) were inspected with 
varying degree of visible defect /thorn, burn etc. / through the nets. Thirteen (18.05\%) mosquito nets were re-treatable but, remaining $9(69.23 \%)$ respondents hadn't re-treated their nets. The major reasons given for none re-treating bed nets were- absence of K-O tablet (retreating kit) was given by 7 $(77.77 \%)$ respondents and2 $(22.23 \%)$ were due to lack of information about the need of re- treatment.

Among total respondents 89 (95.7\%) have information about ITN and the remaining 4 (4.3\%) haven't information about ITN. Among these the assessment done on where they get information about bed net for the first time has showed that $17(19.1 \%)$ of pregnant women's get information about bed net for the first time from community, $65(73.03 \%)$ of pregnant women's from health institution and $7(7.86 \%)$ respondent's get from other sources and 69 (95.8\%) of respondents were got ITN from health institution and 3 $(4.2 \%)$ were got from sale.

Insecticide Treated Nets utilization among pregnant women
The assessment done on utilization of ITNs for the pregnant women in the area has shown that only $51(70.8 \%)$ of pregnant women had self-reported as utilizing bed net the previous night of survey. The direct observation done had shown that only $39(54.1 \%)$ of pregnant women were directly observed while sleeping under bed net. Self-reported utilization of ITNs among pregnant women was over reported on direct observation. The observation of bed nets utilized by pregnant women showed that $19(48.7 \%)$ of utilizes were observed sleeping under properly mounted mosquito net. Similarly, 18 (46.1\%) of pregnant women utilizing net were observed sleeping under bed net with defect and $2(5.12 \%)$ of pregnant women were observed sleeping under bed net which were not treated for the last 12 months. The observation of bed nets utilized by pregnant women showed that $19(37.25 \%)$ of pregnant women's properly utilize mosquito net (Table 2) and (figure 1).

Table 2. Utilization of ITNs among pregnant women over direct observation, Halaba Kulito town, 2016.

\begin{tabular}{lll}
\hline Characteristics & Number & (\%) \\
\hline Have ITNs (n=93) & 72 & 77.4 \\
Pregnant women slept in previous night in the area $(\mathrm{n}=72)$ & 39 & 54.16 \\
Pregnant women sleeping under properly hanged net $(\mathrm{n}=39)$ & 19 & 48.7 \\
Pregnant women sleep under net with defect $(\mathrm{n}=39)$ & 18 & 46.1 \\
Pregnant women sleeping under net which were not retreated $(\mathrm{n}=39)$ & 2 & 5.2 \\
\hline
\end{tabular}

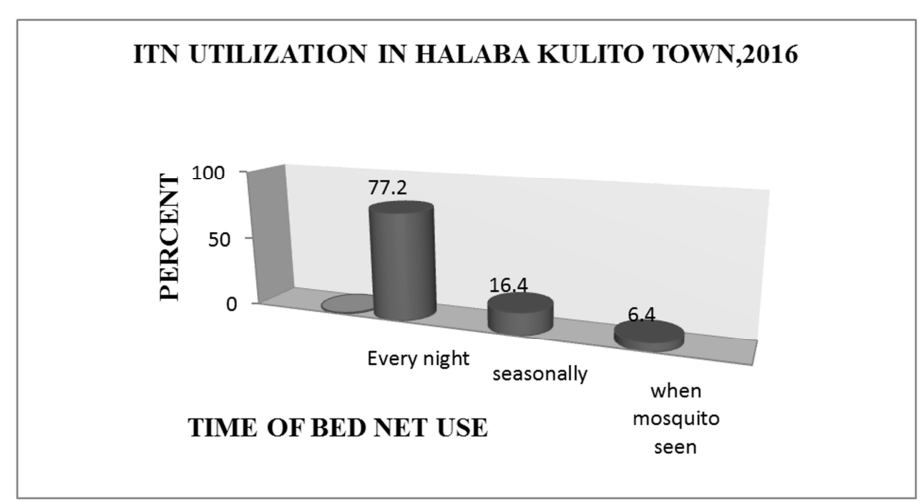

Figure 1. Time of ITN utilization among pregnant women, in Halaba Kulito town, 2016.

Reasons for not-utilizing of ITNs among pregnant women's

Among the reasons for non-utilization, $28.37 \%$ pregnant women not owning bed net and $71.63 \%$ of pregnant women not utilize due to improper utilization of bed net (Table 3 ).

Table 3. Reason for not utilization of ITN among pregnant women's, Halaba kulito town, 2016.

\begin{tabular}{|c|c|c|c|}
\hline S. $\mathbf{N}$ & Variables & Reasons & Percent \\
\hline \multirow[t]{4}{*}{1} & No ITN $(n=21)$ & Expecting for free distribution of bed net by government & 14.9 \\
\hline & & Lack of information & 7 \\
\hline & & Causes discomfort & 5.4 \\
\hline & & Others & 2.7 \\
\hline \multirow[t]{4}{*}{2} & Not using ITNs in the last night $(\mathrm{n}=12)$ & Forgot to mount & 6.4 \\
\hline & & Did not want & 4 \\
\hline & & No mosquito seen in the house & 3 \\
\hline & & Others & 2 \\
\hline \multirow[t]{2}{*}{3} & Slept in bed net with problem $(n=20)$ & Using bed nets having defect & 23.32 \\
\hline & & Sleeping under non treated bed net for the last 12 months & 2.7 \\
\hline \multirow[t]{3}{*}{4} & Not slept under bed net (self-reported) $n=21$ & No comfort & 13.51 \\
\hline & & Cause heat & 8.1 \\
\hline & & Air hunger & 4.05 \\
\hline
\end{tabular}




\subsection{Factors Affecting Ownership of Bed Net and Its Utilization}

The utilization of ITNs was significantly associated with the educational status, bed room condition and time taken to reach health unit of the respondents. The pregnant women's who had primary education were 5.03 times more likely utilizes bed net than no education, $(\mathrm{COR}=5.03,95 \% \mathrm{CI}$, $1.479,120.165)$, $p$-value $=0.021$. The assessment done on bed room condition showed that those pregnant women's who have separated bed room utilizes 5.63 times than who have shared bed room with others $(\mathrm{COR}=5.63,95 \% \mathrm{CI}, 1.61$ 7.265)) $\mathrm{p}$-value $=0.018$ and pregnant women time taken to reach health unit less than $1 \mathrm{hr}$ utilize 4.2 times than those who take greater than $1 \mathrm{hr}$ to reach health center $(\mathrm{COR}=4.2$, 95\% CI, 1.023-19.56) $\mathrm{p}$-value $=0.001$. However, there was no significant association demonstrated from the data between other factors (socio-demographic variables, source of information, ANC follow up and pregnant women utilization of bed net (Table 4).

Table 4. Associated factors affecting utilization of ITN among pregnant women, Halaba Kulito town, 2016.

\begin{tabular}{|c|c|c|c|c|}
\hline \multirow{2}{*}{ Variables } & \multicolumn{2}{|c|}{ Utilized ITN } & \multirow{2}{*}{ OR $(95 \% \mathrm{CI})$} & \multirow{2}{*}{ P-value } \\
\hline & Yes & No & & \\
\hline \multicolumn{5}{|l|}{ Educational status } \\
\hline No education & 3 & 17 & 1 & \\
\hline Primary education & 8 & 9 & $5.03(1.479-120.165)$ & 0.021 \\
\hline Secondary education & 3 & 32 & & \\
\hline More than secondary & 5 & 16 & & \\
\hline \multicolumn{5}{|l|}{ Bed room condition } \\
\hline Shared with others & 3 & 38 & 1 & \\
\hline \multicolumn{5}{|l|}{ Time taken to reach health unit } \\
\hline$<1 \mathrm{hr}$ & 18 & 60 & $4.2(1.023-19.56)$ & 0.001 \\
\hline$\geq 1 \mathrm{hr}$ & 1 & 14 & 1 & \\
\hline
\end{tabular}

\section{Discussion}

The study showed that $72(77.4 \%)$ pregnant women's own at least one bed net. This was higher than the study done Enugu Nigeria [10]. This could be due to high distribution ITN by Halaba woreda health office in collaboration with regional health bearuea. Among 79 pregnant women's which had self-reported as owning bed net, only $7(8.8 \%)$ were missed during direct observation of household's bed net. This was another different finding compared to study done in Burundi, which had shown that $28 \%$ of free distributed bed nets were resold to the neighboring country [11]. The finding of this study is similar to different studies done in Eritrea, Burkina Faso and Ghana $[1,11,12]$. this could be due to lack awareness about the advantage of ITN.

Concerning the factors for non-owning of bed net, $52.38 \%$ was waiting for free distribution through village authority without any attempt to purchase from social market. This figure was higher than the study done in Nigeria. This could be because of lack of awareness on purchasing ITN from social market.

Concerning the re-treatment of bed nets, $69.23 \%$ of retreatable bed nets were reported as none re-treated for last 12 months. This is lower than the study done in Adama woreda [9]. This could be due to close follow up of health extension workers. Another area of concern was the factors given for pregnant women not utilizing bed net the previous night of survey. Similarly to other study done in Ghana forgot to mount the bed net during the night was among the major reasons given [12].

The assessment done on the timing of ITN utilization shows that $78 \%$ every night, which is encouraging compared to the study done in Kenya $50 \%$ [13]. This might be due to the health information dissemination on timing ITN utilization by health workers of Halaba Kulito town.

The survey done on ITN use of pregnant women who slept under ITN the previous night of the survey was (54.1\%). this is $20.1 \%$ higher than the survey done on ITN use of pregnant women who slept under ITN the last night of the survey in Ghana [10]. This is might be due to the dissemination of health information on ITN utilization by the health extension workers.

Concerning the associated factors of ITN utilization, pregnant women's who had primary education were 5.03 times more likely utilizes bed net than no education, $(\mathrm{COR}=5.03,95 \% \mathrm{CI}, 1.479,120.165), \mathrm{p}$-value $=0.021$. This is different with study done in Nigeria and Uganda [10, 12]. This might be due to they have more information about ITN utilization than no education.

The assessment done on bed room condition showed that those pregnant women's who have separated bed room utilizes 5.63 times than who have shared bed room with others $(\mathrm{COR}=5.63,95 \% \mathrm{CI}, 1.61-7.265)) \mathrm{p}$-value $=0.018$. and Time taken to reach health unit less than $1 \mathrm{hr}$ utilize 4.2 times than those who take greater than $1 \mathrm{hr}$ to reach health center $(\mathrm{COR}=4.2,95 \% \mathrm{CI}, 1.023-19.56) \mathrm{p}$-value $=0.001$. This is different from the study done in Uganda [12]. This could be because of social responsibility which cannot make them free and they missed out ITN and vital messages from antenatal 
clinic, which could have an influence over their attitude toward ITN utilization.

The assessment done on marital status of the study participants shows no association with ITN utilization unlike the study done in Kenya which shows married pregnant women's utilize ITN than single/widowed/divorced [13]. This could be due to equal distribution of ITN for all pregnant women's. Concerning the parity of pregnant women's there is between parity ITN utilization in our study while the study done in Southern Tanzania showed that multiparous women's utilize ITN than others [15].

This could be due equal distribution of ITN for all pregnant women's regardless of their parity. Concerning the age of respondent's there is no association between the age of respondent and ITN utilization while the study done in Adama showed that age grouped 18-24 utilizes ITN than other's [9].

In our study there is no association between ANC follow up and ITN utilization while the done in Kenya showed that pregnant women who have gone for ante natal were 1.83 times utilize more likely than those who have not gone for ante natal service [13]. This might be due to health information dissemination by health extension workers of the study area by home to home visit.

\section{Conclusion}

Majority of the pregnant women in Halaba Kulito had not utilized ITN (79.57\%). Only about $20.43 \%$ of the pregnant women had utilized ITN properly. Not having free insecticides treated net and lack of knowledge how to use properly was seen in this study. Expecting free distribution from government, lack of information and causes discomfort was main reason for not owning of ITN. The educational status and housing condition was poor in the study area. The utilization of ITN is significantly associated with time taken to reach health unit, type of bed room condition and level of education but there is no significant association with other factors (socio demographic characteristics, ANC follow up, parity of respondents, source of delivery, number of bed net).

\section{Recommendation}

The free distribution should prioritize vulnerable groups using good opportunity of Antenatal care clinic, household education and regular district level on going survey with great emphasis to Halaba special woreda health office. Halaba special woreda health office can potentially use the existing awareness about cause of malaria and ITNs protection in malaria control program to improve the observed utilization (none compliance) problem. Stakeholders (Ministry of Health and NGOs) should ensure adequate supply of ITN to pregnant women and also sensitize them on the benefits of ITN utilization. Also, leaders should encourage pregnant women to acquire and use ITN as a mean of reducing their vulnerability to malaria disease since they live in housing that offers little protection against mosquitoes.

\section{Limitations of the Study}

This study didn't include proper tacking of pregnant women mosquito net under the mattress or materials used similarly, as mothers go out of bed unpacking the net to open the door for the observers and it may affect proper utilization.

Although, this study has been able to examine ITNs use throughout the entire period of pregnancy, the data collected was based on ability of the women to recall events of the last nine months of pregnancy.

\section{Declarations}

\section{Ethical Approval and Consent to Participant}

Ethical clearance was obtained from Arbaminch University College of Medicine and Health Science department of Midwifery. After approval, formal official letter was written to Halaba Kulito town administrative office to get permission and cooperation. The respondent was informed about the objective, purpose of the study and verbal consent was taken from respondents, and their confidentiality was kept.

\section{Availability of Data and Material}

All related data has been presented within the manuscript. The data set supporting the conclusions of this article is available from the authors on request.

\section{Consent for Publication}

Not applicable.

\section{Competing Interests}

The authors declare that no one have competing interests.

\section{Funding}

Arbaminch University.

\section{Author Contribution}

Both authors have read and approved the manuscript. Both supervised the study design and statistical analysis and contributed to the writing of the manuscript and both authors approved the submitted version of the manuscript.

\section{List of Abbreviations and Acroyms}

ANC: Antenatal Care, DHS: Demographic and Health Survey, ETB: Ethiopian Birr, FMOHE: Federal Ministry of Health of Ethiopia, HH: House Hold, ITNs: Insecticide Treated Nets, MDG: Millennium Development Goal: NGO's -Non-Governmental Organization, RBM: Roll Back Malaria, UNICEF: United Nations children's fund, WHO: World Health Organization 


\section{Acknowledgements}

The authors thank Arbaminch University for approval of ethical clearance, technical and financial support of this study. Then, we would like to thank all study participants who participated in this study for their commitment in responding to our interviews and observations. Lastly, we are indebted to each department health offices for their assistance and permission to undertake the research.

\section{References}

[1] World Health Organization, United Nation Children's Fund and Roll Back Malaria. 2005 World malaria report, WHO, Geneva 2005

[2] Woyessa, A. The elucidation of malaria transmission and its prevalence in highland urban area of Akaki TowN, Addis Ababa University, 2001, PP 120.

[3] Ministry of Health. Malaria diagnosis and treatment guidelines for health workers in Ethiopia, Addis Ababa, 1999

[4] Megha S, Graham B. and Stephen J, ownership and use of insecticides treated nets during pregnancy in sub-saharaAfrica, sing et al malaria journal, 2013, 12 (68) 1-2, 5-6.

[5] Malaria Diagnosis and Treatment Guidelines For Health Workers in Ethiopia: 2nd edition, July 2004 (The Federal Ministry of Health)).

[6] Central Statistics Agency, Ethiopian DHS 2005 preliminary report, CSA, MEASURE DHS, ORC Macro.
[7] Tulu, A. N. Malaria. In: Kloos H and Zein, Z. A., (eds). of health and disease in Ethiopia. Boulder, Westview Press, 1993, Pp. 341-352.

[8] MOH, National health communication strategy, October 2004.

[9] Bogale F. assessment of insecticides treated nets among childrens under five years of age and pregnant womens of Adamaworeda, oromia regional state, Ethiopia.

[10] Prosper ouA, ChristianI, insecticides treated nets possession and utilization among pregnant womens in Enugu Nigeria, journal of natural sciences research 2013, 1 (2) 45-48.

[11] Yared L, Ayalew T, Tefera B, Kora T, ownership and use of treated bed nets in urban communities of Assosa zone, western Ethiopia, Ethiopia J. Health sci. 2008, 17 (4) 205-210.

[12] Obol JH1, 4, Ononge S2, Orach CG3 Utilization of insecticide treated nets among pregnant women in Gulu: a post conflict district in northern Uganda.

[13] The American Society of Tropical medicine and hygiene, community -wide effects of permethrin treated nets on child mortality and malaria morbidity in western in western Kenya, AM. J. Trop. Med. hyg. 68 (s. 4), 2003, pp 121-127.

[14] Thomas M. Factors associated with utilization of insecticides treated nets among pregnant women in northern regions of Namibia January 2014 (28-3).

[15] Kabanywanyi AM, Macarthur JR, Stolk WA, Habbema JD, Mshinda H, Bloland PB, et al. Malaria in pregnant women in an area with sustained high coverage of insecticide-treated bed nets. Malar J. 2008; 7: 133. 